\title{
Conservation Laws of Three-Dimensional Perfect Plasticity Equations under von Mises Yield Criterion
}

\author{
S. I. Senashov ${ }^{1}$ and A. Yakhno ${ }^{2}$ \\ ${ }^{1}$ Siberian State Aerospace University, 31 Krasnoyarsky Rabochy Avenue, Krasnoyarsk 660014, Russia \\ 2 Departamento de Matemáticas, CUCEI, Universidad de Guadalajara, Boulevard Marcelino García Barragán 1421, \\ 44430 Guadalajara, JAL, Mexico
}

Correspondence should be addressed to A. Yakhno; alexander.yakhno@cucei.udg.mx

Received 4 July 2013; Revised 22 September 2013; Accepted 26 September 2013

Academic Editor: A. F. Cheviakov

Copyright (c) 2013 S. I. Senashov and A. Yakhno. This is an open access article distributed under the Creative Commons Attribution License, which permits unrestricted use, distribution, and reproduction in any medium, provided the original work is properly cited.

For the first time the conservation laws for von Mises plasticity equations in three dimensions as well as for plane stress equations are given. In the plane case conservation laws are used to construct characteristics for the Cauchy problem. For the system of the plane strain, the conservation laws are used to solve the free boundary problem for any convex smooth contour loaded with constant normal and zero tangential stresses.

\section{Introduction}

Conservation laws are becoming one of the most important tools for studying and solving differential equations. Such significance was gained after the article by Noether [1]. In this paper it was shown that there is a close relationship between the so-called Noether's symmetries and conservation laws. Namely, the conservation law corresponds to each such symmetry.

Later it was shown that such a relationship exists only for the equations derived from the variational principle. For other equations the so-called operator of universal linearization should be constructed. And then it is necessary to consider the kernel of its (formally) adjoint operator [2, 3]. In particular, it permits consistently to construct the conservation laws of plasticity equations and moreover to use them to solve the main boundary value problems of the plane theory of perfect plasticity $[4,5]$.

Let us give some basic definitions of the theory of conservation laws due to [2]. Let us consider a system

$$
\mathscr{F}: F_{r}\left(\bar{x}, \bar{u}, \bar{p}_{s}\right)=0, \quad r=1, \ldots, m,
$$

of $m$ differential equations for $m$ unknown functions $\bar{u}=$ $\left(u^{1}, \ldots, u^{m}\right)$ and its derivatives $\bar{p}_{s}=\left\{\overline{u^{\alpha}}, \overline{u^{\alpha}}, \ldots, \overline{u^{\alpha}}\right\}$ up to the order $s$ with respect to $\bar{x}=\left(x_{1}, \ldots, x_{n}\right)$ independent variables $(\alpha=1, \ldots, m)$.

A conserved current is an $n$-dimensional vector function $\bar{A}=\left(A_{1}, \ldots, A_{n}\right)$ with components (fluxes) depending on $\bar{x}$, $\bar{u}, \overline{u^{\alpha}}, \ldots, \overline{u^{\alpha}}, \ldots$, which satisfy the following relation for any solution of $\mathscr{F}$ :

$$
\frac{\partial A_{i}}{\partial x_{i}}=\square_{r}\left(F_{r}\right)
$$

where $\square_{r}$ are some scalar differential operators. The above relation (2) is called conservation law of system $\mathscr{F}$. Let us note that all conserved currents are considered to be equivalence classes with respect to trivial currents (for which $\operatorname{div} \bar{A}=0$ for any form of $\bar{u}$ ). Hereafter the summation over repeating indices is assumed.

There follows (see [4] and cited bibliography) the following theorem.

Theorem 1. Let system $\mathscr{F}$ of differential equations (1) be derived from a variational principle and admit the following point Noether's symmetry $(i=1, \ldots, n ; \alpha=1, \ldots, m)$ :

$$
X=\xi^{j} \frac{\partial}{\partial x_{j}}+\eta^{\alpha} \frac{\partial}{\partial u^{\alpha}} .
$$


Then the ith component of conserved current has the following form:

$$
A_{i}^{(X)}=\eta^{\alpha} \frac{\partial L}{\partial u_{i}^{\alpha}}+\xi^{i} L-\xi^{j} u_{j}^{\alpha} \frac{\partial L}{\partial u_{i}^{\alpha}}
$$

where $L\left(\bar{x}, \bar{u}, \overline{u^{\alpha}}\right)$ is the density of the corresponding Lagrangian, $u_{i}^{\alpha}=\partial u^{\alpha} / \partial x_{i}$.

Let us recall [2] that a functional

$$
\mathscr{L}=\int L\left(\bar{x}, \bar{u}, \overline{u^{\alpha}}, \overline{u^{\alpha}}, \ldots, \overline{u_{s}^{\alpha}}, \ldots\right) d \bar{x}
$$

is called a Lagrangian (or an action or a variational functional), and function $L$ is called the density of $\mathscr{L}$.

\section{Conservation Laws of Three-Dimensional Plasticity Equations}

Let us consider [6] the system of perfect isotropic plasticity in the three-dimensional stationary case which describes the state of the plastic flow of an incompressible plastic medium and consists of three equilibrium equations:

$$
\frac{\partial p}{\partial x_{i}}=\frac{\partial s_{i j}}{\partial x_{j}}
$$

the von Mises yield criterion which is defined when the medium achieves a plastic state

$$
s_{i j} s_{i j}=2 k^{2} \text {, }
$$

the incompressibility equation

$$
\frac{\partial u^{i}}{\partial x_{i}}=0
$$

and the relation between components $s_{i j}=\sigma_{i j}+p \delta_{i j}$ of the deviatoric stress and components $e_{i j}$ of the symmetric strainrate tensor:

$$
s_{i j}=\frac{\lambda}{2}\left(\frac{\partial u^{i}}{\partial x_{j}}+\frac{\partial u^{j}}{\partial x_{i}}\right)=\lambda e_{i j},
$$

where $\left\{x_{1}, x_{2}, x_{3}\right\}$ is a Cartesian coordinate system, $p=$ $-\sigma_{i i} / 3$ is the hydrostatic pressure, $u^{i}$ are the components of the velocity vector, $k$ is the yield point, $\lambda=\lambda\left(x_{1}, x_{2}, x_{3}\right)$ is a positive function defined by yield criterion, $\sigma_{i j}$ are components of stress tensor, $\delta_{i j}$ is the Kronecker delta, and $i$, $j=1,2,3$. Eliminating $s_{i j}$ and $\lambda$ from (6)-(9) we come to four nonlinear equations for functions $p, u^{1}, u^{2}, u^{3}$ only:

$$
F_{i}: \frac{\partial p}{\partial x_{i}}-k \frac{\partial}{\partial x_{j}}\left(\frac{u_{j}^{i}+u_{i}^{j}}{\sqrt{2 e_{i j} e_{i j}}}\right)=0, \quad F_{4}: u_{i}^{i}=0 \text {, }
$$

where $u_{i}^{j}=\partial u^{j} / \partial x_{i}$
System (10) admits Lie algebra $L_{15}$ of point transformations spanned by the following generators [7] $(i=1,2,3)$ :

$$
\begin{aligned}
& X_{i}=\frac{\partial}{\partial x_{i}}, \quad Y_{i}=\frac{\partial}{\partial u^{i}}, \\
& S=\frac{\partial}{\partial p}, \quad N=x_{i} \frac{\partial}{\partial x_{i}}, \quad M=u^{i} \frac{\partial}{\partial u^{i}} \\
& Z_{1}=x_{2} \frac{\partial}{\partial x_{3}}-x_{3} \frac{\partial}{\partial x_{2}}+u^{2} \frac{\partial}{\partial u^{3}}-u^{3} \frac{\partial}{\partial u^{2}}, \\
& T_{1}=x_{2} \frac{\partial}{\partial u^{3}}-x_{3} \frac{\partial}{\partial u^{2}} .
\end{aligned}
$$

Generators $Z_{2}, T_{2}, Z_{3}$, and $T_{3}$ are obtained from $Z_{1}, T_{1}$ by cyclic permutation of indices. The group of point transformations is generated by the following monoparametric subgroups:

$$
\begin{gathered}
x_{i}^{\prime}=x_{i}+a_{i}, \quad u^{i^{\prime}}=u^{i}+b_{i}, \quad p^{\prime}=p+c, \\
x_{i}^{\prime}=x_{i} \exp \left(a_{i+3}\right), \quad u^{i^{\prime}}=u^{i} \exp \left(b_{i+3}\right), \\
x_{2}^{\prime}=x_{2} \cos \varphi_{1}+x_{3} \sin \varphi_{1}, \quad x_{3}^{\prime}=-x_{2} \sin \varphi_{1}+x_{3} \cos \varphi_{1}, \\
u^{2^{\prime}}=u^{2} \cos \varphi_{1}+u^{3} \sin \varphi_{1}, \\
u^{3^{\prime}}=-u^{2} \sin \varphi_{1}+u^{3} \cos \varphi_{1}, \\
u^{2^{\prime}}=u^{2}+d_{1} x_{3}, \quad u^{3^{\prime}}=u^{3}-d_{1} x_{2},
\end{gathered}
$$

where $a_{i}, b_{i}, \varphi_{i}$, and $d_{i}, c$ are group parameters.

Transformations (12) are translations with respect to $x_{i}$, $u^{i}, p$; (13) are scaling on $x_{i}, u^{i}$; (14) are three rotations in the planes $x_{1} O x_{2}, x_{2} O x_{3}$, and $x_{3} O x_{1}$ and there are three rigid displacements (15).

System (10) can be deduced from the Lagrangian $(\bar{u}=$ $\left.\left(u^{1}, u^{2}, u^{3}, p\right)\right)$ :

$$
\mathscr{L}=\iiint L\left(\bar{x}, \bar{u}, \underset{1}{u^{\alpha}}\right) d x_{1} d x_{2} d x_{3},
$$

where the density of the Lagrangian has the form

$$
L=\sigma_{i j} e_{i j}=s_{i j} e_{i j}-p e_{i i}=k \sqrt{2 e_{i j} e_{i j}}-p\left(u_{1}^{1}+u_{2}^{2}+u_{3}^{3}\right) \text {, }
$$

and it represents the velocity of dissipation of mechanical energy in elemental volume.

It is easy to verify that (10) are the Euler-Lagrange equations corresponding to $\mathscr{L}$ which look as follows:

$$
\begin{aligned}
& F_{i}: \frac{\partial L}{\partial u_{i}}-\frac{\partial}{\partial x_{j}}\left(\frac{\partial L}{\partial u_{j}^{i}}\right)=0, \\
& F_{4}: \frac{\partial L}{\partial p}-\frac{\partial}{\partial x_{j}}\left(\frac{\partial L}{\partial p_{j}}\right)=0,
\end{aligned}
$$

where $p_{i}=\partial p / \partial x_{i}$ 
Let us note that the von Mises yield criterion (7) was obtained from a variation principle: actual stresses in plastic state maximize the velocity of surface forces work [8] for an incompressible medium. For such a medium quantity $\sqrt{2 e_{i j} e_{i j}}$ is called the intensity of the share strain rate [6].

For system (10) one can construct the conservation laws with the help of Noether's symmetries. It is well known that Noether's symmetries are a subalgebra of Lie algebra of point symmetries admitted by $\mathscr{F}$. It is sufficient from symmetries (11) to determine the ones that do not change the density (17). To verify if the given point symmetry $X$ is a Noether's one, let us use the known condition of Lagrangian density invariance [9]

$$
\underset{1}{X}(L)+L D_{i}\left(\xi^{i}\right)=0
$$

Here $\underset{1}{X}$ is the first prolongation of $X$ of the form (3), where $\xi^{j}$ are the coefficients of $\partial / \partial x_{j}, \eta^{\alpha}$ are the coefficients of $\partial / \partial u^{\alpha}$, and $\eta^{4}$ is the coefficient of $\partial / \partial p ; D_{i}$ is the total derivative operator along $x_{i}$ :

$$
D_{i}(f)=\frac{\partial}{\partial x_{i}}+\frac{\partial f}{\partial x_{i}} \frac{\partial}{\partial f}+\frac{\partial^{2} f}{\partial x_{i} \partial x_{j}} \frac{\partial}{\partial\left(\partial f / \partial x_{j}\right)}+\cdots .
$$

The direct verification of (19) for symmetries (11) shows that $X_{i}, Y_{i}, Z_{i}$, and $T_{i}$ are point Noether's symmetries of (17). Using Theorem 1 and taking into account that

$$
\frac{\partial L}{\partial u_{j}^{i}}=-p \delta_{i j}+s_{i j}=\sigma_{i j}
$$

one can obtain the fluxes of conserved current, corresponding to generator $X_{1}(j=1,2,3)$

$$
\begin{aligned}
& A_{1}^{\left(X_{1}\right)}=L+p u_{1}^{1}-u_{1}^{j} s_{1 j}=L-u_{1}^{j} \sigma_{1 j}, \\
& A_{2}^{\left(X_{1}\right)}=-u_{1}^{j} \sigma_{2 j}, \quad A_{3}^{\left(X_{1}\right)}=-u_{1}^{j} \sigma_{3 j},
\end{aligned}
$$

and the conservation law represents the conservation of generalized momentum along the $x_{1}$-axis and reflects the homogeneity of the medium.

For generator $Z_{1}$, the components of the conserved current look as follows:

$$
\begin{aligned}
A_{1}^{\left(Z_{1}\right)}= & \left(x_{3} u_{2}^{1}-x_{2} u_{3}^{1}\right)\left(s_{11}-p\right)+\left(x_{3} u_{2}^{2}-x_{2} u_{3}^{2}-u^{3}\right) s_{12} \\
& +\left(x_{3} u_{2}^{3}-x_{2} u_{3}^{3}+u^{2}\right) s_{13}, \\
A_{2}^{\left(Z_{1}\right)}= & \left(x_{3} u_{2}^{1}-x_{2} u_{3}^{1}\right) s_{21}+\left(x_{3} u_{2}^{2}-x_{2} u_{3}^{2}-u^{3}\right)\left(s_{22}-p\right) \\
& +\left(x_{3} u_{2}^{3}-x_{2} u_{3}^{3}+u^{2}\right) s_{23}-x_{3} L, \\
A_{3}^{\left(Z_{1}\right)}= & \left(x_{3} u_{2}^{1}-x_{2} u_{3}^{1}\right) s_{31}+\left(x_{3} u_{2}^{2}-x_{2} u_{3}^{2}-u^{3}\right) s_{32} \\
& +\left(x_{3} u_{2}^{3}-x_{2} u_{3}^{3}+u^{2}\right)\left(s_{33}-p\right)+x_{2} L,
\end{aligned}
$$

and represent the conservation law of angular momentum.
For $Y_{1}$ we have the following fluxes:

$$
A_{1}^{\left(Y_{1}\right)}=-p+s_{11}=\sigma_{11}, \quad A_{2}^{\left(Y_{1}\right)}=\sigma_{12}, \quad A_{3}^{\left(Y_{1}\right)}=\sigma_{13},
$$

and the conservation law is just the first equilibrium equation from (6) which was obtained from conservation of momentum. By analogy, the conservation laws for generators $Y_{2}$ and $Y_{3}$ correspond to the second and the third equilibrium equations.

Generator $T_{1}$ produces

$$
\begin{gathered}
A_{1}^{\left(Y_{1}\right)}=-x_{3} \sigma_{12}+x_{2} \sigma_{13}, \quad A_{2}^{\left(Y_{1}\right)}=-x_{3} \sigma_{22}+x_{2} \sigma_{23}, \\
A_{3}^{\left(Y_{1}\right)}=-x_{3} \sigma_{23}+x_{2} \sigma_{33} .
\end{gathered}
$$

By analogy, one can construct the conserved currents for $X_{2,3}$, $Z_{2,3}, T_{2,3}$.

It is easy to see that the linear combination $P=N-2 M$ is a point Noether's symmetry of (17). Then, the components of corresponding conserved current from $(4)$ are $(j=1,2,3)$ :

$$
\begin{aligned}
A_{1}^{(P)}= & x_{1} L-\left(2 u^{1}+x_{j} u_{j}^{1}\right) \sigma_{11}-\left(2 u^{2}+x_{j} u_{j}^{2}\right) \sigma_{12} \\
& -\left(2 u^{3}+x_{j} u_{j}^{3}\right) \sigma_{13}, \\
A_{2}^{(P)}= & x_{2} L-\left(2 u^{1}+x_{j} u_{j}^{1}\right) \sigma_{21}-\left(2 u^{2}+x_{j} u_{j}^{2}\right) \sigma_{22} \\
& -\left(2 u^{3}+x_{j} u_{j}^{3}\right) \sigma_{23}, \\
A_{3}^{(P)}= & x_{3} L-\left(2 u^{1}+x_{j} u_{j}^{1}\right) \sigma_{31}-\left(2 u^{2}+x_{j} u_{j}^{2}\right) \sigma_{32} \\
& -\left(2 u^{3}+x_{j} u_{j}^{3}\right) \sigma_{33} .
\end{aligned}
$$

Let us note that generator $S$ is not a point Noether's symmetry of (17), because relation (19) for $S$ does not vanish:

$$
\underset{1}{S}(L)+L D_{i}\left(\xi^{i}\right)=-\left(u_{1}^{1}+u_{2}^{2}+u_{3}^{3}\right)=D_{i}\left(B_{i}\right),
$$

where $B_{i}=u^{i}$. In such a case, Noether's symmetry is called the symmetry of divergence type and it produces [9] the conservation law of the form $D_{i}\left(C_{i}\right)=0$, where $C_{i}=A_{i}^{(X)}-B_{i}$ and $A_{i}^{(X)}$ are given by (4). So, for $S$ we obtain $A_{i}^{(S)}=0$ and $C_{i}=-u^{i}$ which represents the conservation law of the mass given by (8).

For Navier-Stokes equations the complete set of conservation laws was found in [10]. Due to relative analogy of plastic flow system and Navier-Stokes equations it is quite difficult to expect conservation laws other than those specified here.

\section{Conservation Laws of Plane Stress Equations}

A plane stress state is approximately achieved in a thin lamina deformed under the action of forces which lie in its median 
plane. Equations for stresses in a Cartesian plane $x O y$ can be written as follows [6]:

$$
\begin{aligned}
& F_{1}=\frac{\partial \varphi}{\partial x}+\frac{\sqrt{3}}{2} \sin 2 \varphi \frac{\partial \omega}{\partial x}-\frac{1}{2}(\sqrt{3} \cos 2 \varphi+\cot \omega) \frac{\partial \omega}{\partial y}=0 \\
& F_{2}=\frac{\partial \varphi}{\partial y}-\frac{1}{2}(\sqrt{3} \cos 2 \varphi-\cot \omega) \frac{\partial \omega}{\partial x}-\frac{\sqrt{3}}{2} \sin 2 \varphi \frac{\partial \omega}{\partial y}=0
\end{aligned}
$$

where the angle $\omega(x, y)$ is connected with the value of the mean pressure $\sigma: \cos \omega=\sigma \sqrt{3} /(2 k)$ and $\varphi(x, y)$ is the angle between the first principal direction of stress tensor and the $x$-axis.

To construct the conservation laws let us use the method proposed in [2]. Firstly it is necessary to construct the matrix differential operator of the universal linearization $l_{\mathscr{F}}$ (or the Fréchet derivative of $\mathscr{F}$ [11]). Then considering the formally adjoint operator $l_{\mathscr{F}}^{*}$ one needs to solve the equation

$$
l_{\mathscr{F}}^{*}(\Psi)=0,
$$

restricted to the solutions of (28). Here $\Psi=\left(\Psi^{1}, \Psi^{2}\right)$ is called the generating function of the conservation law. Let us recall that operator $l_{\mathscr{F}}$ has the form of matrix

$$
l_{\mathscr{F}}=\left\|l_{i j}\right\|=\left\|\frac{\partial F_{i}}{\partial u_{\sigma}^{j}} D_{\sigma}\right\|
$$

where $u_{\sigma}^{j}=\partial^{|\sigma|} u^{j} / \partial x_{1}^{i_{1}} \cdots \partial x_{n}^{i_{n}}, \sigma=\left(i_{1}, \ldots, i_{n}\right)$ being a multiindex, $|\sigma|=i_{1}+\cdots+i_{n} \leqslant s$, and $D_{\sigma}=D_{i_{1}} \circ \cdots \circ D_{i_{n}}$. And if $l_{\mathscr{F}}=a_{\sigma} D_{\sigma}=\left\|l_{i j}\right\|$, then $l_{\mathscr{F}}^{*}=(-1)^{|\sigma|} D_{\sigma} \circ a_{\sigma}=\left\|l_{j i}^{*}\right\|$.

After some manipulations (29) takes the following form:

$$
\begin{aligned}
D_{x}( & \left.\Psi^{1}\right)-\sqrt{3}\left(\cos 2 \varphi \frac{\partial \omega}{\partial x}+\sin 2 \varphi \frac{\partial \omega}{\partial y}\right) \Psi^{1} \\
& +D_{y}\left(\Psi^{2}\right)-\sqrt{3}\left(\sin 2 \varphi \frac{\partial \omega}{\partial x}-\cos 2 \varphi \frac{\partial \omega}{\partial y}\right) \Psi^{2}=0 \\
2 \sqrt{3} & \left(\cos 2 \varphi \frac{\partial \varphi}{\partial x}+\sin 2 \varphi \frac{\partial \varphi}{\partial y}\right) \Psi^{1}+\sqrt{3} \sin 2 \varphi D_{x}\left(\Psi^{1}\right) \\
& -(\sqrt{3} \cos 2 \varphi+\cot \omega) D_{y}\left(\Psi^{1}\right) \\
& +2 \sqrt{3}\left(\sin 2 \varphi \frac{\partial \varphi}{\partial x}-\cos 2 \varphi \frac{\partial \varphi}{\partial y}\right) \Psi^{2} \\
& -\sqrt{3} \sin 2 \varphi D_{y}\left(\Psi^{2}\right) \\
& -(\sqrt{3} \cos 2 \varphi-\cot \omega) D_{x}\left(\Psi^{2}\right)=0
\end{aligned}
$$

where the derivatives, for example, $\partial \varphi / \partial x$ and $\partial \omega / \partial x$, should be changed by the corresponding expressions due to (28). It is clear that (31) have an infinite number of solutions. Let us consider one of them, namely, the one of the form $\Psi^{1}=$ $\Psi^{1}(\varphi, \omega), \Psi^{2}=\Psi^{2}(\varphi, \omega)$.

Let us note that it is possible to determine other solutions of (29), but as far as the authors know for the conservation laws depending on the derivative there is no application to solve any practical problems.

Relations (31) should be valid for any solution of system (28), and therefore the coefficients of derivatives $\partial \omega / \partial x$, $\partial \omega / \partial y$ are equal to zero identically. It gives two linear equations to determine the components of conserved current $\bar{A}=(A(\varphi, \omega), B(\varphi, \omega))$ :

$$
\begin{aligned}
& \frac{\partial A(\varphi, \omega)}{\partial \omega}-\frac{\sqrt{3}}{2} \frac{\partial A(\varphi, \omega)}{\partial \varphi} \sin 2 \varphi \\
& +\frac{1}{2} \frac{\partial B(\varphi, \omega)}{\partial \varphi}(\sqrt{3} \cos 2 \varphi-\cot \omega)=0, \\
& \frac{\partial B(\varphi, \omega)}{\partial \omega}+\frac{\sqrt{3}}{2} \frac{\partial B(\varphi, \omega)}{\partial \varphi} \sin 2 \varphi \\
& +\frac{1}{2} \frac{\partial A(\varphi, \omega)}{\partial \varphi}(\sqrt{3} \cos 2 \varphi+\cot \omega)=0
\end{aligned}
$$

corresponding to generating function $\Psi$ with $\Psi^{1}=\partial A / \partial \varphi$, $\Psi^{2}=\partial B / \partial \varphi$. It is easy to verify, substituting $\left(\Psi^{1}, \Psi^{2}\right)$ in $(31)$ and taking into account the above system and its differential consequences. In such a case, the differential operators $\square_{r}$ from (2) are just two functions:

$$
\square_{1}=\square_{1}^{*}=\frac{\partial A}{\partial \varphi}, \quad \square_{2}=\square_{2}^{*}=\frac{\partial B}{\partial \varphi} .
$$

Finally, the following theorem is valid.

Theorem 2. The system of plane stress state admits infinite series of conservation laws.

System (28) has two distinct families of real characteristic curves in the hyperbolic region (if $4 \cos ^{2} \omega<3$ ), defined by equations [6]:

$$
\frac{d y}{d x}=\tan (\varphi-\psi), \quad \frac{d y}{d x}=\tan (\varphi+\psi)
$$

with relations along characteristics

$$
\Omega-\varphi=\text { const., } \quad \Omega+\varphi=\text { const. }
$$

where

$$
\begin{gathered}
\Omega=-\frac{1}{2} \int_{\pi / 6}^{\omega} \frac{\sqrt{3-4 \cos ^{2} \omega}}{\sin \omega} d \omega, \quad \psi=\frac{\pi}{2}-\frac{1}{2} \arccos \frac{\cot \omega}{\sqrt{3}}, \\
\frac{\pi}{6}<\omega<\frac{5 \pi}{6},
\end{gathered}
$$

where $2 \psi$ is the angle of intersection of characteristic curves. Let us introduce two new unknown functions $\xi$ and $\eta$ (characteristic coordinates or Riemann invariants) in such a way: $\Omega-\varphi=\xi, \Omega+\varphi=\eta$.

Then system (28) takes the form

$$
\frac{\partial \xi}{\partial x}+\tan (\varphi-\psi) \frac{\partial \xi}{\partial y}=0, \quad \frac{\partial \eta}{\partial x}+\tan (\varphi+\psi) \frac{\partial \eta}{\partial y}=0
$$


Finally, for the components of conserved current we have the following linear equations:

$$
\frac{\partial B}{\partial \xi}-\tan (\varphi-\psi) \frac{\partial A}{\partial \xi}=0, \quad \frac{\partial B}{\partial \eta}-\tan (\varphi+\psi) \frac{\partial A}{\partial \eta}=0 .
$$

Let functions

$$
\left.\xi\right|_{P Q}=\xi_{0}(x, y),\left.\quad \eta\right|_{P Q}=\eta_{0}(x, y)
$$

be given along the smooth curve $P Q$ (see Figure 1). Curve $P Q$ is not a characteristic and it intersects with each characteristic only once. Let $M\left(m_{1}, m_{2}\right)$ be a point of intersection of characteristics $\xi=\xi_{1}=$ const. going from point $P\left(p_{1}, p_{2}\right)$ and $\eta=\eta_{1}=$ const. going from point $Q\left(q_{1}, q_{2}\right)$. Then it is necessary to define a solution of the Cauchy problem (37), (39) in curvilinear triangle PQM [12].

Let us determine the coordinates of point $M$. Taking an integral over the closed path $P Q M$ which due to the Green theorem is equal to zero we have

$$
\begin{aligned}
\oint_{P Q M} A d y-B d x \\
=\int_{\xi=\xi_{1}} A d y-B d x+\int_{\eta=\eta_{1}} A d y-B d x \\
\quad+\int_{P Q} A d y-B d x=0 .
\end{aligned}
$$

Then, by integrating parts we obtain

$$
\begin{aligned}
& \int_{\xi=\xi_{1}} A d y-B d x \\
&=\int_{M P}(A \tan (\varphi-\psi)-B) d x \\
&=\left.x(A \tan (\varphi-\psi)-B)\right|_{x=m_{1}} ^{x=p_{1}} \\
&-\int_{\xi=\xi_{1}} x d(A \tan (\varphi-\psi)-B), \\
& \int_{\eta=\eta_{1}} A d y-B d x \\
&=\int_{Q M}(A \tan (\varphi+\psi)-B) d x \\
&=\left.x(A \tan (\varphi+\psi)-B)\right|_{x=q_{1}} ^{x=m_{1}} \\
&-\int_{\eta=\eta_{1}} x d(A \tan (\varphi+\psi)-B) .
\end{aligned}
$$

Without loss of generality let us take

$$
A \tan (\varphi-\psi)-B=1, \quad \text { along } \xi=\xi_{1} .
$$

Then we have

$$
\int_{\xi=\xi_{1}} A d y-B d x=p_{1}-m_{1}
$$

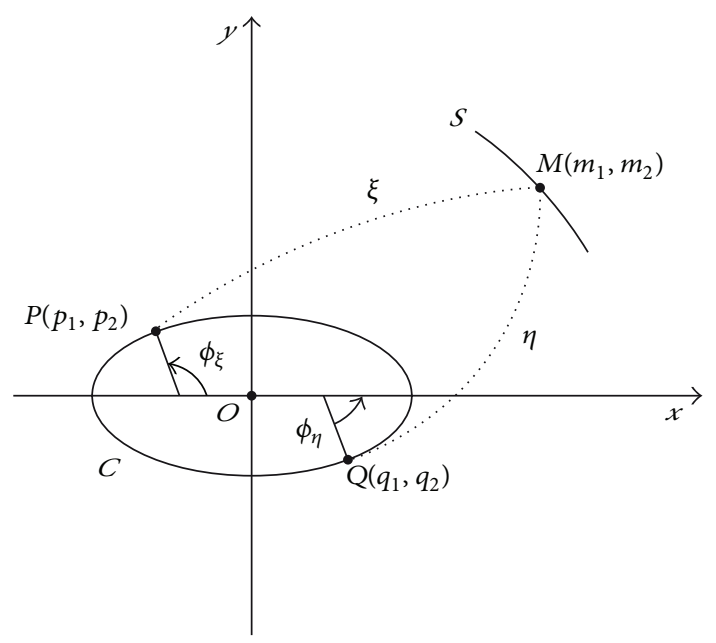

Figure 1: The Cauchy problem.

By analogy

$$
\int_{\eta=\eta_{1}} A d y-B d x=0
$$

if we put

$$
A \tan (\varphi+\psi)-B=0, \quad \text { along } \eta=\eta_{1} .
$$

Finally, from (40) we have

$$
m_{1}=p_{1}+\int_{P Q} A d y-B d x
$$

where components of the conserved current $A, B$ are defined from the linear system (38) and boundary conditions (42), (45).

For the second coordinate of $M$ we have

$$
\begin{array}{rl}
\int_{\xi=\xi_{1}} A d y-B d x & \\
= & \int_{M P}(A-B \cot (\varphi-\psi)) d y \\
= & \left.y(A-B \cot (\varphi-\psi))\right|_{y=m_{2}} ^{y=p_{2}} \\
& -\int_{\xi=\xi_{1}} y d(A-B \cot (\varphi-\psi)), \\
\int_{\eta=\eta_{1}} & A d y-B d x \\
= & \int_{Q M}(A-B \cot (\varphi+\psi)) d y \\
= & \left.y(A-B \cot (\varphi+\psi))\right|_{y=q_{2}} ^{y=m_{2}} \\
& -\int_{\eta=\eta_{1}} y d(A-B \cot (\varphi+\psi)) .
\end{array}
$$

Taking

$$
A-B \cot (\varphi-\psi)=1, \quad \text { along } \xi=\xi_{1},
$$


we obtain

$$
\begin{gathered}
\int_{\xi=\xi_{1}} A d y-B d x=p_{2}-m_{2}, \\
\int_{\eta=\eta_{1}} A d y-B d x=0
\end{gathered}
$$

if we take

$$
A-B \cot (\varphi+\psi)=0, \quad \text { along } \eta=\eta_{1}
$$

Finally, from (40) one can determine the second coordinate of point $M$

$$
m_{2}=p_{2}+\int_{P Q} A d y-B d x
$$

where components of conserved current $A, B$ are solutions of (38) with boundary conditions (48), (50).

The solution of the aforementioned problems for conserved currents gives the solutions of the Cauchy problem for the initial system, because at point $M$ one can reconstruct the values of functions $\xi$ and $\eta$ from boundary conditions (39) and, finally, functions $\Omega$ and $\varphi$.

In such a way it is possible to determine the families of characteristic curves for the system of the plane stress. These curves do not coincide with the so-called slip lines (lines where the tangent stress achieved its maximum value) as in the case of the plane strain system considered later. But principal directions of the stress tensor bisect the angles between characteristic curves. That is why it is possible to reconstruct slip lines from the known characteristic curves field.

\section{Conservation Laws of Plane Strain Equations}

Let us consider the system of perfect plane plasticity with Tresca-Saint-Venant-Mises yield condition [6]:

$$
\begin{aligned}
& \frac{\partial \sigma}{\partial x}-2 k\left(\frac{\partial \theta}{\partial x} \cos 2 \theta+\frac{\partial \theta}{\partial y} \sin 2 \theta\right)=0 \\
& \frac{\partial \sigma}{\partial y}-2 k\left(\frac{\partial \theta}{\partial x} \sin 2 \theta-\frac{\partial \theta}{\partial y} \cos 2 \theta\right)=0
\end{aligned}
$$

where $\sigma$ is the hydrostatic pressure, $\theta+\pi / 4$ is the angle between the first principal direction of stress tensor and the $x$-axis, and $k$ is the plasticity constant. System (52) describes the plain strain state of a plastic deformed medium. In such a state the displacements of particles of the homogeneous and isotropic body are parallel to $x \mathrm{O} y$ plane and are independent of the third coordinate $z$.

Quasilinear system (52) is of the hyperbolic type and characteristics are given by the following equations:

$$
\frac{d y}{d x}=\tan \theta, \quad \frac{d y}{d x}=-\cot \theta .
$$

Along corresponding characteristics the following relations are valid:

$$
\frac{\sigma}{2 k}-\theta=\text { const., } \quad \frac{\sigma}{2 k}+\theta=\text { const. }
$$

The complete set of conservation laws of (52) as a solution of (29) is described in detail in [13]. In [5], the conserved currents depending only on $\sigma, \theta$ are applied to solve main boundary problems and are similar to (38) when $\varphi=\theta+\pi / 4$, $\psi=\pi / 4$. Thus, the solution of problem (38), (42), (45) has a form

$$
A=2 \frac{\partial \rho}{\partial \xi} \cos \theta-\rho \sin \theta, \quad B=2 \frac{\partial \rho}{\partial \xi} \sin \theta+\rho \cos \theta
$$

where function $\rho(\xi, \eta)$ looks like the following:

$$
\begin{aligned}
\rho(\xi, \eta)= & R\left(\xi, \xi_{1}, \eta, \eta_{1}\right) \cos \left(\frac{\eta_{1}-\xi_{1}}{2}\right) \\
& -\frac{1}{2} \int_{\eta_{1}}^{\eta} R\left(\xi, \xi_{1}, \eta, \tau\right) \sin \left(\frac{\tau-\xi_{1}}{2}\right) d \tau
\end{aligned}
$$

Accordingly, the solution of problem (38), (48), (50) is

$$
\begin{aligned}
\rho(\xi, \eta)= & R\left(\xi, \xi_{1}, \eta, \eta_{1}\right) \sin \left(\frac{\eta_{1}-\xi_{1}}{2}\right) \\
& +\frac{1}{2} \int_{\eta_{1}}^{\eta} R\left(\xi, \xi_{1}, \eta, \tau\right) \cos \left(\frac{\tau-\xi_{1}}{2}\right) d \tau
\end{aligned}
$$

where $R\left(\xi, \xi_{1}, \eta, \eta_{1}\right)=I_{0}\left(\sqrt{\left(\xi-\xi_{1}\right)\left(\eta-\eta_{1}\right)}\right)$ is the modified Bessel function of the first kind of a zero order having the following properties:

$$
\begin{aligned}
& I_{0}(0)=1, \quad I_{0}^{\prime}(0)=0, \\
& \frac{d I_{0}(z)}{d z}=I_{1}(z), \quad \frac{d I_{1}(z)}{d z}=I_{0}(z)-\frac{I_{1}(z)}{z} .
\end{aligned}
$$

Let us consider the problem of a free boundary. Let the constant normal $\sigma_{n}$ and null tangent components $\tau_{n}$ of stresses be given along a smooth contour $C$ of some convex cavity (see Figure 1), situated in an infinite plastic deformed medium:

$$
\sigma_{n}^{C}=-p, \quad \tau_{n}^{C}=0 .
$$

It is necessary to determine the free boundary $S$ along which

$$
\sigma_{n}^{S}=\tau_{n}^{S}=0
$$

The relation between functions $\sigma, \theta$ of (52) and normal components $\sigma_{n}, \tau_{n}$ looks as follows [6]:

$$
\sigma_{n}=\sigma-k \sin 2\left(\theta-\phi^{C}\right), \quad \tau_{n}=k \cos 2\left(\theta-\phi^{C}\right)
$$

where $\phi^{C}$ is the angle between the normal to the contour $C$ and $x$-axis. 


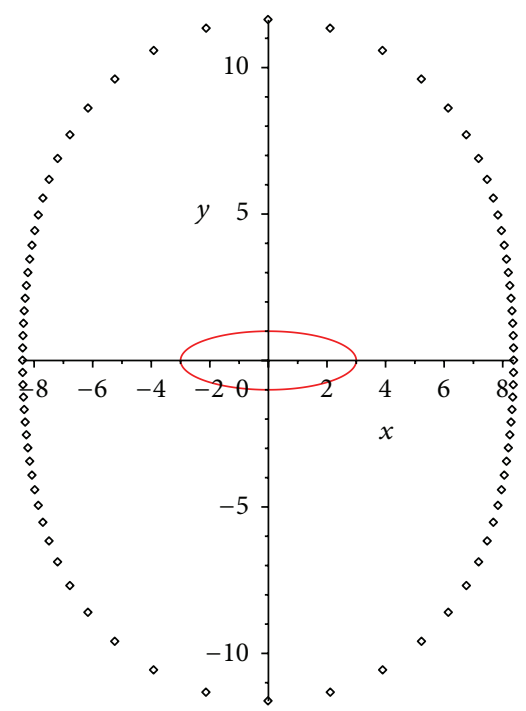

Figure 2: Free boundary for the contour $\left(x^{2} / 9\right)+y^{2}=1$.

Substituting (59) in (61), we have along $C(m \in \mathbb{Z})$

$$
\theta^{C}=\phi^{C} \pm \frac{\pi}{4}+m \pi, \quad \sigma^{C}=-p \pm k=\text { const. }
$$

Let us draw two characteristics

$$
\xi=\frac{\sigma^{C}}{2 k}-\theta_{\xi}^{C}, \quad \eta=\frac{\sigma^{C}}{2 k}+\theta_{\eta}^{C}
$$

where $\theta_{\xi}^{C}, \theta_{\eta}^{C}$ are the values of $\theta^{C}$ at points $P, Q \in C$, respectively.

Applying the above method we define the coordinates of $M$ where we have

$$
\begin{gathered}
\sigma^{M}=k(\eta+\xi)=\sigma^{C}+k\left(\phi_{\eta}^{C}-\phi_{\xi}^{C}\right), \\
\theta^{M}=\frac{\eta-\xi}{2}=\frac{\phi_{\eta}^{C}+\phi_{\xi}^{C}}{2} \pm \frac{\pi}{4}+m \pi .
\end{gathered}
$$

On the other hand, given (60) we obtain

$$
\sigma^{M}= \pm k, \quad \theta^{M}=\phi^{M} \pm \frac{\pi}{4}+m \pi
$$

Equating the right-hand sides of (64), (65) we have

$$
p=k\left(\phi_{\eta}^{C}-\phi_{\xi}^{C}\right), \quad \phi^{M}=\frac{\phi_{\eta}^{C}+\phi_{\xi}^{C}}{2} .
$$

As a particular example, let us consider contour $C$ in the form of an ellipse (Figure 1). Equality (66) is valid, if $\phi_{\eta}^{C}-$ $\phi_{\xi}^{C}=m \pi$. Changing the coordinates of points $P, Q$, one can find curve $S$, which will be the boundary free of stresses. In Figure 2 some points of the free boundary for the contour $C$ : $x^{2} / 9+y^{2}=1$ are given for $p=k \pi$.

\section{Conclusions}

For all point symmetries of a three-dimensional perfect plasticity system with the von Mises yield criterion the corresponding conservation laws are calculated. Some of them (conservation of the mass and of the impulse) are in the basis of this system; however, there are new conservation laws.

Recently a rigid plasticity constitutive model with the linear kinematic hardening has been analyzed in [14]. Focusing on planar simple shear the constitutive functions were classified according to continuous symmetry groups. It will be interesting to consider the conservation laws of the obtained systems.

How two linear systems for the components of conserved currents for the system of plane stress state can be used to determine its characteristic curves is shown.

For the perfect strain plane plasticity with Tresca-SaintVenant-Mises yield condition, the problem of a free boundary for arbitrary convex cavity situated in the infinite plastic deformed medium is solved with the use of the corresponding conservation laws.

\section{Acknowledgments}

The work was supported by the Ministry of Education and Science of the Russian Federation (Project 1.3720.2011) to S. I. Senashov and by PRO-SNI 2013-UDG to A. Yakhno.

\section{References}

[1] E. Noether, "Invariante variations probleme," Nachrichten der Königlichen Gesellschaft der Wissenschaften zu Göttingen, pp. 235-257, 1918.

[2] I. S. Krasil'shchik and A. M. Vinogradov, Eds., Symmetries and Conservation Laws for Differential Equations of Mathematical Physics, vol. 182 of Translations of Mathematical Monographs, American Mathematical Society, Providence, RI, USA, 1999.

[3] A. M. Vinogradov, "Local symmetries and conservation laws," Acta Applicandae Mathematicae, vol. 2, no. 1, pp. 21-78, 1984.

[4] S. I. Senashov and A. Yakhno, Aplicación de Simetrías y Leyes de Conservación a la Resolución de Ecuaciones Diferenciales de Mecánica, Universidad de Guadalajara, 2008, (Spanish).

[5] S. I. Senashov and A. Yakhno, "Conservation laws, hodograph transformation and boundary value problems of plane plasticity," SIGMA, vol. 8, no. 071, 2012.

[6] L. M. Kachanov, Fundamentals of the Theory of Plasticity, Dover Books on Engineering, Dover, 2004.

[7] S. I. Senashov, "Invariant solutions of a three-dimensional ideal plasticity problem," Journal of Applied Mechanics and Technical Physics, vol. 21, no. 3, pp. 417-420, 1980.

[8] A. M. Freudenthal and H. Geiringer, "The mathematical theories of the inelastic continuum," in Elasticity and Plasticity, S. Flügge, Ed., vol. 3-6 of Encyclopedia of Physics, pp. 229-433, Springer, Berlin, Germany, 1958.

[9] L. V. Ovsiannikov, Group Analysis of Differential Equations, Academic Press, New York, NY, USA, 1982.

[10] V. N. Gusyatnikova and V. A. Yumaguzhin, "Symmetries and conservation laws of Navier-Stokes equations," Acta Applicandae Mathematicae, vol. 15, no. 1-2, pp. 65-81, 1989. 
[11] P. J. Olver, Applications of Lie Groups to Differential Equations, vol. 107 of Graduate Texts in Mathematics, Springer, New York, NY, USA, 1986.

[12] B. L. Roždestvenskiı̌ and N. N. Janenko, Systems of Quasilinear Equations and Their Applications to Gas Dynamics, vol. 55 of Translations of Mathematical Monographs, American Mathematical Society, Providence, RI, USA, 1983, Translated from the Second Russian Edition by J. R. Schulenberger.

[13] S. I. Senashov and A. M. Vinogradov, "Symmetries and conservation laws of 2-dimensional ideal plasticity," Proceedings of the Edinburgh Mathematical Society, vol. 31, no. 3, pp. 415-439, 1988.

[14] A. F. Cheviakov, J. F. Ganghoffer, and R. Rahouadj, "Finite strain plasticity models revealed by symmetries and integrating factors: the case of Dafalias spin model," International Journal of Plasticity, vol. 44, pp. 47-67, 2013. 


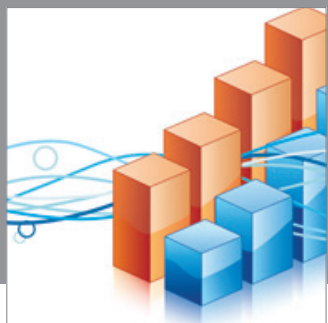

Advances in

Operations Research

mansans

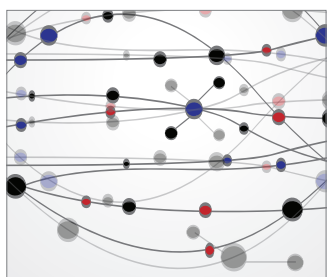

The Scientific World Journal
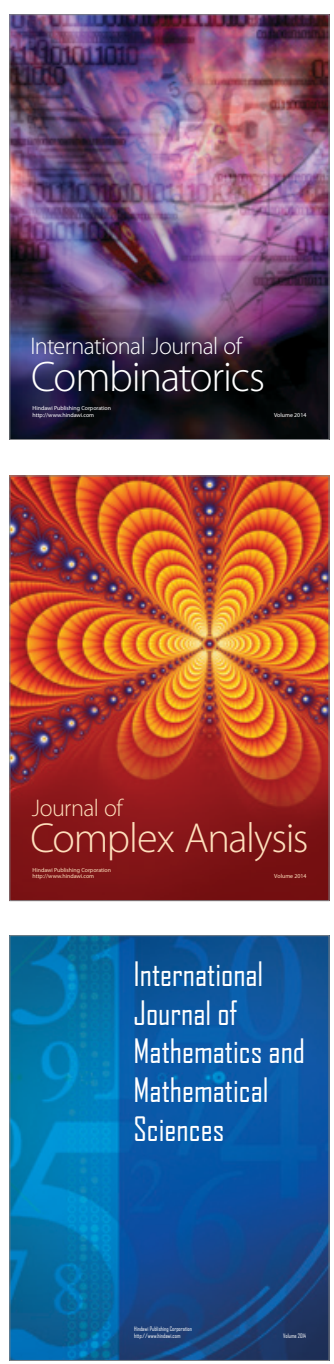
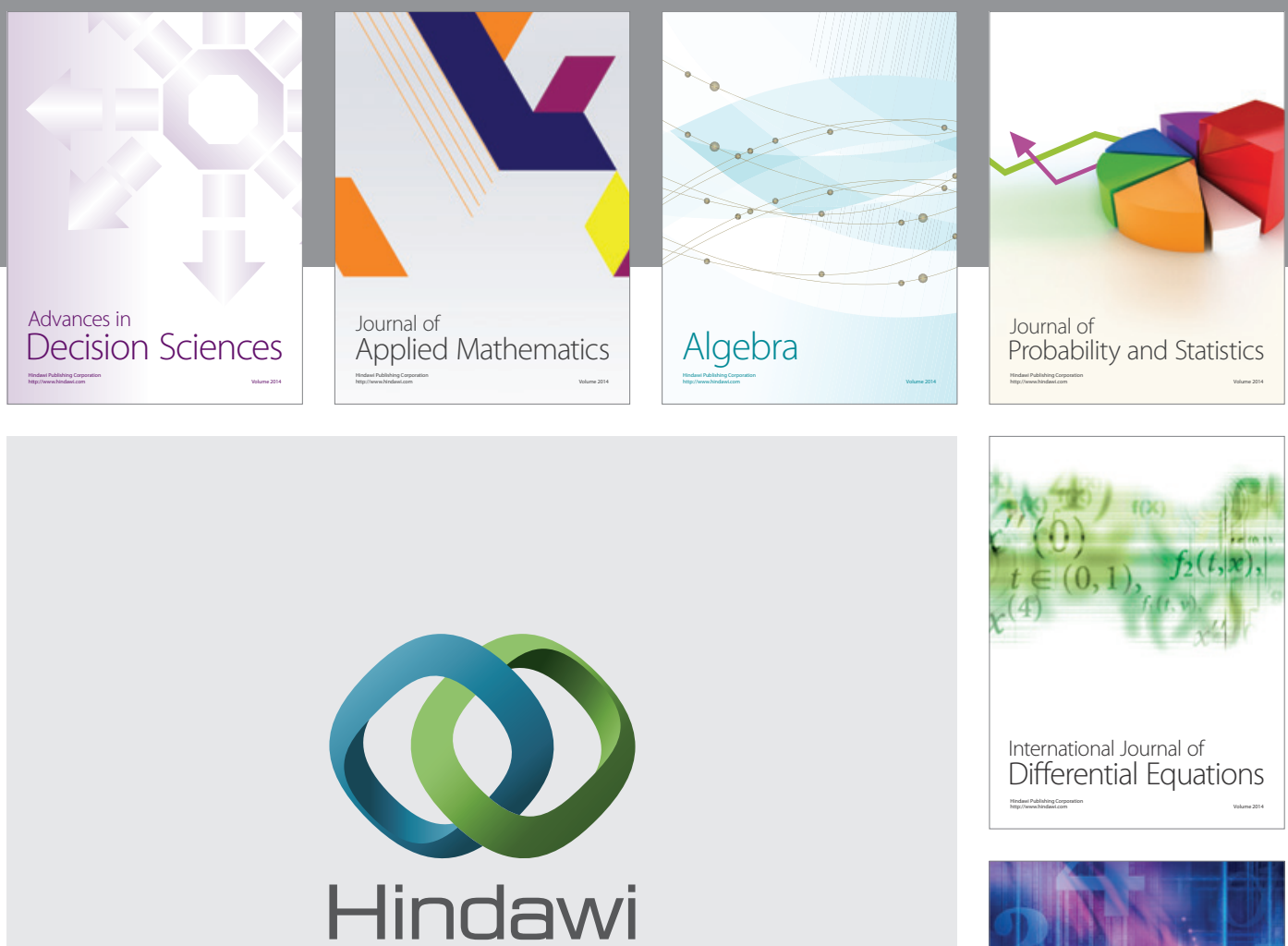

Submit your manuscripts at http://www.hindawi.com
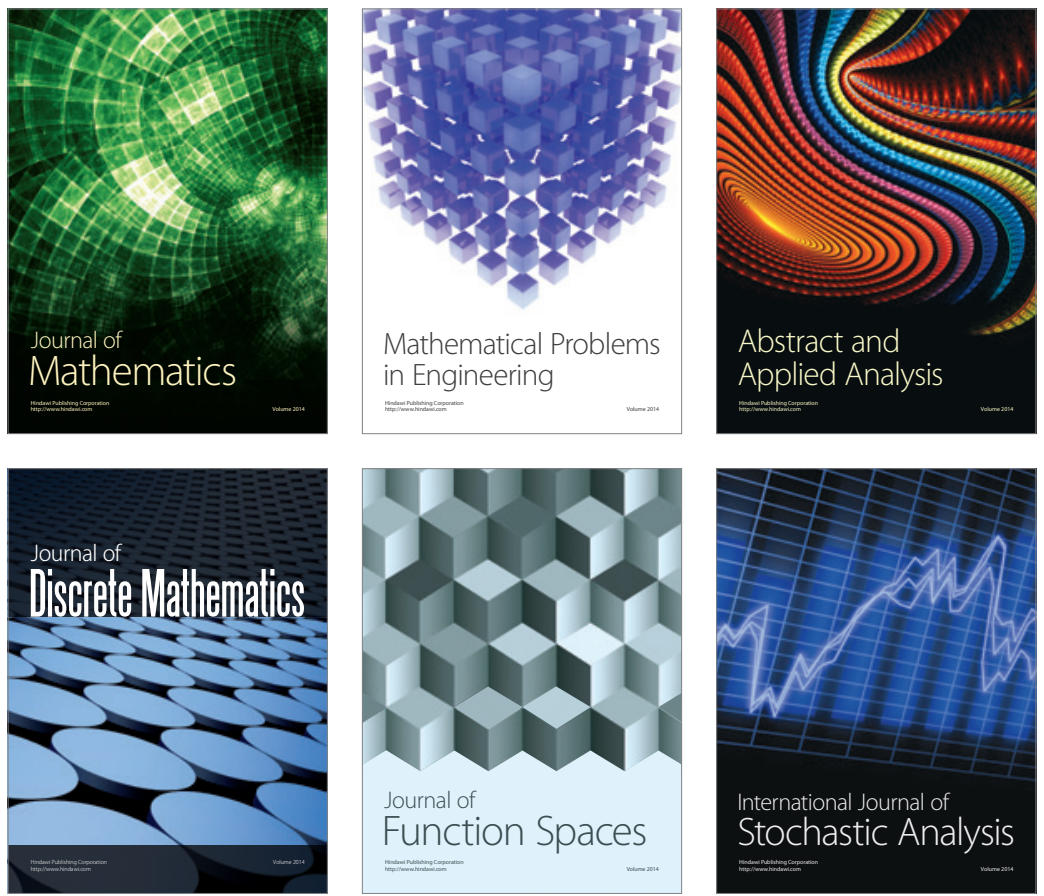

Journal of

Function Spaces

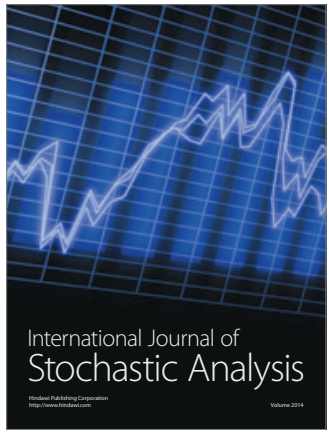

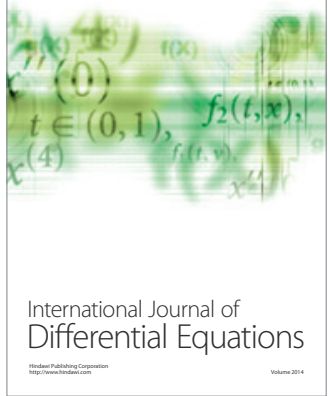
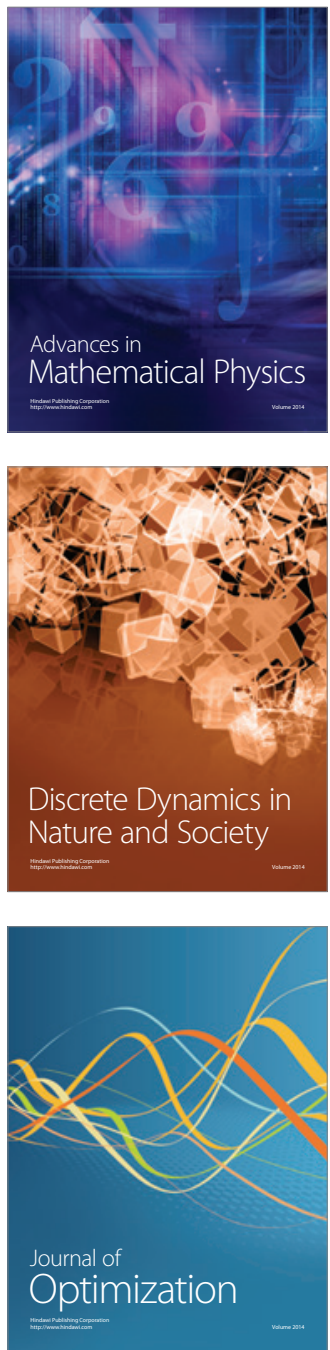